\title{
TIME
}

B R I L L

Timing \& Time Perception 4 (2016) 207-222

$\overline{\text { brill.com/time }}$

\section{Effect of Modified-Release Methylphenidate on Cognition in Children with ADHD: Evidence from a Temporal Preparation Task}

\author{
Antonino Vallesi ${ }^{1,2, *}$, Elisa D'Agati ${ }^{3}$, Clementina Grelloni ${ }^{3}$, Augusto Pasini ${ }^{3}$, \\ Giovanni Mazzotta ${ }^{4}$ and Paolo Curatolo ${ }^{3}$ \\ ${ }^{1}$ Dipartimento di Neuroscienze, Università degli Studi di Padova, Via Giustiniani, \\ 5, 35128 Padua, Italy \\ ${ }^{2}$ Centro di Neuroscienze, Università degli Studi di Padova, Padua, Italy \\ ${ }^{3}$ Dipartimento di Medicina dei Sistemi, Policlinico Tor Vergata, Rome, Italy \\ ${ }^{4}$ Dipartimento di Salute Mentale, ASL2, Università degli studi di Perugia, Perugia, Italy \\ Received 22 February 2016; accepted 18 May 2016
}

\begin{abstract}
ADHD is associated with various cognitive deficits, including general performance decrements and specific impairments, for instance in temporal processing. However, time preparation under uncertain conditions has been under-investigated in this population. We aimed at filling this gap. We administered a variable foreperiod paradigm to children with ADHD before and after a onemonth treatment with modified-release methylphenidate. Age-matched ADHD children with no treatment and healthy children were also tested as control groups with the same time-schedule. Children with ADHD had general performance deficits (longer and more variable response times), which disappeared in the experimental group after pharmacological intervention. Moreover, ADHD children showed a marked dependency on sequential foreperiod effects (i.e., slower responses for longer preceding foreperiods), especially at short current foreperiods, which were not modulated by the pharmacological treatment. In conclusion, the present findings show that methylphenidate enhances general motor processes rather than more specific time preparation processes, some of which appear deviant in ADHD.
\end{abstract}

Keywords

Children, ADHD, methylphenidate, performance variability, time processing, response preparation, foreperiod effect, sequential effects

* To whom correspondence should be addressed. E-mail: antonino.vallesi@unipd.it 


\section{Introduction}

Attention-deficit/hyperactivity disorder (ADHD) is one of the most prevalent developmental disorders characterized by symptoms of inattention, hyperactivity and impulsivity (American Psychiatric Association, 2013). ADHD is also associated with neurocognitive impairments (e.g., Vallesi et al., 2013a; see Sergeant et al., 2002, for a review). Some studies highlight basic information processing problems in ADHD as shown by response slowing and increased response time (RT) variability (Johnson et al., 2008; Klein et al., 2006; Kuntsi et al., 2003; Scheres et al., 2003).

More specific deficits include problems in inhibitory control (Kramer et al., 2001) and spatial attention orienting, which allows attention to be allocated to and manipulated within certain regions of visual space, independently of whether attention orienting is triggered strategically through a symbolic central cue (McDonald et al., 1999) or automatically through a changing peripheral cue (Perchet et al., 2001). Therefore, it seems likely that ADHD may affect the effectiveness of visuo-motor preparation, including the ability to use warning signals to effectively orient attention not only in space but also in time.

It is also known that ADHD children have problems with time processing in general, which may result in time underestimation, suggesting that they have an abnormally fast internal clock with respect to controls (Luman et al., 2009; Toplak et al., 2006), although these deficits may not be observed in musical contexts (Carrer, 2015). Time processing deficits in ADHD may be related to problems with preparation and planning and with hyperactive and impulsive behavior (Oosterlaan et al., 2005; Sergeant et al., 2002). A recent review shows that ADHD patients are consistently impaired in three main time-processing domains: motor timing, perceptual timing and temporal foresight, with the deficits being associated with dysfunctions in the fronto-striato-cerebellar and fronto-parietal networks that mediate time-processing (Noreika et al., 2013).

An underinvestigated aspect of the general time-processing framework in ADHD is implicit time preparation. This ability has been traditionally studied by manipulating the waiting time between a warning stimulus and a target, the so-called foreperiod (FP; Woodrow, 1914). A small number of previous studies specifically investigating temporal preparation in ADHD used an implicit time preparation design with a fixed preparatory interval (foreperiod) administration (Hurks et al., 2005; Leth-Steensen et al., 2000). One of these studies showed that ADHD children have problems preparing a response with relatively long (1000 ms) but not with short (100 ms) fixed FPs (Hurks et al., 2005).

Preparatory strategies, however, may change dramatically as a function of how the FP is manipulated (Niemi \& Näätänen, 1981). The variable FP administration, especially when FPs of different length are administered randomly and equiprobably, has been shown to give rise to both a current trial FP effect (i.e., shorter RTs for 
longer current FPs) and sequential effects (i.e., longer RTs for longer previous FPs). Thus, it remains to be investigated whether ADHD children have problems with temporal preparation in a variable FP context, and in particular with processes linked to sequential effects, which are consistently believed to be automatic (Los \& Van den Heuvel, 2001; Los et al., 2014; Vallesi \& Shallice, 2007) and probably motoric (Vallesi et al., 2007a) in nature, or with dissociable mechanisms underlying the variable FP effect. The latter has been shown to be related, anatomically, to the functionality of the lateral prefrontal cortex (Triviño et al., 2010; Vallesi \& Shallice, 2007; Vallesi et al., 2007a, b) and, cognitively, with a more strategic, controlled process which monitors the changing conditional probability of stimulus occurrence in time (e.g., Niemi \& Näätänen, 1981; Vallesi \& Shallice, 2007; Vallesi et al., 2013b). Indeed, the two effects have been dissociated as a function of taskdemands, with dual-task manipulations affecting more the variable FP effect than sequential effects (Vallesi et al., 2014), as well as in terms of their developmental trajectories, with sequential effects emerging earlier than the variable FP effect (Vallesi \& Shallice, 2007).

From a functional-anatomical point of view, ADHD is associated with dysfunction and anatomical abnormalities in the fronto-striatal circuitry (Cubillo et al., 2012; Curatolo et al., 2009), which, especially in the right hemisphere, correlates with response inhibition deficits in ADHD (Curatolo et al., 2010). A catecholamine dysfunction is also reported in ADHD children (Curatolo et al., 2009; Spencer et al., 2005).

Methylphenidate (MPH) is an effective and well-established treatment for ADHD that has been shown to modulate dopamine and noradrenaline signaling by blocking the respective monoamine transporters, and increasing extracellular dopamine levels in the striatum, with improvements in motivation and reward (Taylor et al., 2004; Banaschewski et al., 2006). It also increases binding of dopamine and the potentiation of dopamine neurotransmission, by diminishing the availability of D2 and D3 receptors, thus reducing inattention and impulsivity. During cognitive tasks MPH increases cerebral blood flow in the prefrontal cortex in ADHD patients (Schweitzer et al., 2004). The MPH efficacy and effectiveness in reducing inattentiveness and impulsiveness is confirmed by numerous placebo-controlled randomized control trials, with an effect size on hyperactivity symptoms in the range 0.8-1.1, and a response rate of at least 70\% (Banaschewski et al., 2006; NICE, 2008).

New-generation formulations of MPH have been developed recently, which have a long-acting profile, since they combine both immediate-release (IR) and modified-release (MR) mechanisms (González et al., 2002; Pelham et al., 2001), therefore eliminating the requirement for extra daily doses (Pelham et al., 2011). A recent quantitative review (Coghill et al., 2014), mostly including studies using single dose MPH-IR, demonstrated that MPH-IR also improves various cognitive processes in ADHD, including executive and non-executive aspects of working 
memory, response inhibition, reaction time (RT) and RT variability. The aim of designing a system-controlled release of a drug is to obtain a formulation that releases the active substance at a rate necessary to achieve and maintain a constant concentration in the blood. Little is known about the effects of new-generation formulations of MPH-MR on cognition.

A goal of the present work was to fill the gap present in the literature regarding the relationship between ADHD and implicit temporal preparation in a variable FP context, and to gain insight into the cognitive effects of MPH-MR in the time preparation domain. In particular, we administered a variable FP task to children with ADHD before and after a one-month pharmacological intervention with MPH-MR. We also recruited and tested with the same modalities another group of age- and sex-matched ADHD children with no pharmacological intervention and a group of healthy control children.

Generally, we expected to replicate the classical finding of slower and more variable absolute RTs in ADHD as obtained with a time preparation RT task. A more specific prediction would be that ADHD children are only influenced by automatic preparation processes, as reflected by sequential FP effects, if they are less able to reach an optimal preparation level with longer FPs in a variable FP context. This hypothesized pattern would be similar to what happens with younger (4-5 years old) children (Vallesi \& Shallice, 2007). The latter prediction also derives from the literature that highlights ADHD-related abnormalities in brain regions (e.g., Casey et al., 1997), as these overlap with the (right) dorsolateral prefrontal cortex recruited for the implementation of the preparatory strategies involved in this paradigm (e.g., Triviño et al., 2010; Vallesi et al., 2007a). In addition, the observation that ADHD children show problems in attentional orienting, as evidenced by using visuo-spatial attention tasks (McDonald et al., 1999; Perchet et al., 2001), also provides support for this predictions. Alternatively, it could be that the already reported deficits in attentional orienting are limited to the visuospatial domain and do not generalize to (implicit) temporal processing.

From a pharmacological point of view, we generally expected to replicate previous findings that MPH intervention would improve performance by accelerating RTs and making them less variable (Coghill et al., 2014; cf. Johnson et al., 2008). More specifically, we wanted to understand whether the pharmacological therapy would improve strategic time preparation processes in ADHD by increasing the variable FP effect and/or by making it less susceptible to automatic sequential effects.

\section{Method}

\subsection{Participants}

Three samples of children were recruited for the present study (see Table 1 for details). The three samples were composed of: 14 children with ADHD who underwent pharmacological intervention 
Table 1.

Clinical and demographic characteristics of our sample of participants at first evaluation.

\begin{tabular}{lccc}
\hline & $\begin{array}{c}\text { ADHD in treatment } \\
\text { with MPH }\end{array}$ & $\begin{array}{c}\text { ADHD without MPH } \\
\text { treatment }\end{array}$ & $\begin{array}{c}\text { Healthy } \\
\text { subjects }\end{array}$ \\
\hline$N$ & 14 & 17 & 14 \\
Sex M/F & $13 / 1$ & $14 / 3$ & $9 / 5$ \\
Mean age in years (range) & $10.8(7-15)$ & $10(6-15)$ & $11(6-14)$ \\
Mean T score Conners' Parents (mean \pm SD) & & \\
Inattention & $72 \pm 8.2$ & $70 \pm 9.7$ & $50 \pm 4.5$ \\
Hyperactivity & $78 \pm 9.3$ & $73 \pm 8.7$ & $46 \pm 4.3$ \\
ADHD index & $76 \pm 8.5$ & $73 \pm 9.3$ & $56 \pm 4$ \\
\hline
\end{tabular}

with methylphenidate therapy; 17 control children with ADHD who did not undergo any pharmacological intervention; and 14 healthy control children. No significant age difference emerged across the groups (for all, $p>0.05$ ). All patients with ADHD were consecutive referrals from the Child Psychiatry Unit of "Tor Vergata" University, Rome. For all of them the ADHD diagnosis was made in accordance with DSM-IV-TR criteria (American Psychiatry Association, 2000). The Conners' Parents Rating Scale (CPRS), the Conners' Teachers Rating Scale (CTRS; Conners, 2007), when possible, and the ADHD Rating Scale-IV were used to support the diagnosis and to define the ADHD subtype. To ensure a homogenous sample, the Kiddie Schedule of Affective Disorders (Kaufman et al., 2004) screening interviews were used to investigate the presence of ADHD during childhood and to exclude other psychiatric comorbidities. Inclusion criteria were: diagnosis of ADHD combined-type, no mental retardation, no brain trauma, no neurological diseases or physical impairment, no comorbid mental disorders (investigated using K-SADS), except for possible learning disabilities (LD). ADHD children were evaluated for learning abilities with the "Battery for the Diagnosis of Developmental Dyslexia" (Sartori, Job, \& Tressoldi, 1995), and the "Battery for the Diagnosis of Developmental Dyscalculia" (Biancardi \& Nicoletti, 2004). Children who received a standardized score below the cut-off for their school grade were given a diagnosis of dyslexia and/or dyscalculia.

Eight participants with ADHD additionally suffered from mixed LD. None of the ADHD diagnosed children had a previous history of stimulant treatment. All the pharmacological treatments were indicated by a child psychiatrist. The non-pharmacological ADHD group included children whose parents refused a pharmacological treatment or children for whom the pharmacological treatment was not an option due to heart problems. For patients with ADHD who started the pharmacological treatment with modified release MPH at the first visit, health history (including vital signs, height, and weight), demographic data and neurological history were checked. They started the pharmacological treatment at an initial dose of $10 \mathrm{mg} /$ day. In the subsequent two visits the neurological assessment was performed, including vital signs, weight, and height, questioning for the presence of any adverse effects, or concurrent treatments and verifying compliance with the treatment. After one month of pharmacological treatment the effective dose ranged from $0.3 \mathrm{mg}$ to $1.5 \mathrm{mg} / \mathrm{kg} /$ day taken as a single dose in the morning. In all ADHD patients the Conners' scale for parents and teachers was completed before starting drug treatment and one month after to verify the improvement of ADHD symptoms. 
Healthy controls were recruited from local schools in Rome. None of them had a history of neurological/psychiatric disease or LD documented by interviewing the children's parents. A potential diagnosis of ADHD, according to the DSM-IV-TR criteria, was excluded in all control participants using the ADHD Rating Scale-IV and CPRS with parents. At the time of testing, no participant was taking medications known to affect the central nervous system. All participants had normal or corrected-to-normal vision. We assume that there were no marked socio-demographic differences between the patients and controls given that all subjects attended public schools and lived in the same area of Rome (although this was not explicitly tested). All subjects enrolled in the study were tested twice a month apart in the late morning.

The study procedure was approved by the Child Psychiatry and Neurology Institute Ethical Committee of Tor Vergata University, Rome and was in accordance with the 1964 Helsinki declaration and its later amendments or comparable ethical standards. Before testing each child, a parent/legal guardian signed a written informed consent form.

\subsection{Materials}

The experiment was conducted on a Toshiba Satellite 1410-604 portable notebook. Responses were collected from the notebook's keyboard. Participants' sight was kept at a distance of about $50-60 \mathrm{~cm}$ from the center of the computer screen, with their dominant hand index finger resting on the spacebar. Auditory Warning Stimulus (WS) was presented at a comfortable level.

All visual stimuli were presented against a black background. A centrally presented cross, consisting of two yellow crossed bars $1.0 \times 0.5 \mathrm{~cm}$ in size, served as the fixation stimulus (FS). The WS was a $1500 \mathrm{~Hz}$ pure tone, presented for $50 \mathrm{~ms}$. The Imperative Stimulus (IS) was a downward pointing white arrow $2 \mathrm{~cm}$ long that consisted of a $1.5 \times 1 \mathrm{~cm}$ bar attached to a $0.5 \mathrm{~cm}$ arrowhead with a maximum width of $2 \mathrm{~cm}$ (note that the stimuli are very similar to those used previously by Los \& Van Den Heuvel, 2001, and by Vallesi \& Shallice, 2007). The FP interval between the warning and the IS could last 1, 2 or $3 \mathrm{~s}$. Each session consisted of 120 trials, where the three FPs were randomly intermixed.

Task. A trial started with the presentation of the FS together with the WS. The onset of the WS marked the beginning of the Foreperiod (FP). When the FP expired, the FS disappeared, and the IS appeared at the center of the screen. Participants were instructed to respond as fast as possible only to the IS by pressing the spacebar. The IS disappeared upon response key press. After a blank interval of $2000 \mathrm{~ms}$, a new trial started.

\subsection{Design and Procedure}

The within-subject independent variables included FP (1, 2 or $3 \mathrm{~s}$ ), session (two sessions with about a one-month interval). For the experimental ADHD group, the second session was administered after the pharmacological treatment with MPH-MR.

Three practice trials were given before the real test, in order to briefly familiarize participants with the procedure and allow the experimenter to check whether the instructions were clear. After the first 60 trials of the test phase, participants received a rest break during which they were encouraged to relax as needed. The break ended when the participants pressed the spacebar and the second and last block of trials started.

\subsection{Data Analysis}

Trials on which the Reaction Time (RT) was beyond the 200-2000 ms range were discarded from further analyses. Mean RTs and standard deviations were analyzed separately though ANOVAs with group (ADHD with pharmacological treatment between sessions, ADHD without treatment, control children), as the between subject factor, and session (first, second), previous FP (1, 2, 3 s) and current FP (1, 2, $3 \mathrm{~s})$, as the within-subject factors. For the significant effects, a post-hoc Tukey tests were 
performed in order to further investigate more specific paired comparisons. Planned comparisons were used to investigate more complex comparisons. Early responses (spacebar press during the FP) were also collected and analyzed in a separate ANOVA.

\section{Results}

\subsection{Reaction Times (RTs)}

Mean RTs are plotted in Fig. 1. There was a significant group main effect $[F(2$, $\left.42)=4.2, p=0.022, \eta_{\mathrm{p}}^{2}=0.17\right]$. Planned comparisons showed that the ADHD group with no intervention had (across the two sessions) longer RTs than both of the other two groups $(p<0.001)$. Also the ADHD group with pharmacological intervention had longer RTs than the healthy control group $(p<0.001)$. The main effects of the current FP $\left[F(2,42)=59.56, p<0.0001, \eta_{\mathrm{p}}{ }^{2}=0.58\right]$ and preceding FP $\left[F(2,42)=61.56, p<0.0001, \eta_{\mathrm{p}}^{2}=0.59\right]$ were also significant. These effects were qualified by a preceding by current FP interaction $[F(4,168)=16.8$, $\left.p<0.0001, \eta_{\mathrm{p}}{ }^{2}=0.28\right]$, which showed the classical asymmetric sequential FP effects (strongest for short FPs). A current FP by group interaction $[F(4,84)=3.77$, $\left.p<0.007, \eta_{\mathrm{p}}{ }^{2}=0.15\right]$ showed that, contrary to the original hypothesis, the two ADHD groups had a more pronounced variable FP effect than controls, as also demonstrated by planned comparisons contrasting differential RTs for shortest and longest current FPs in each pair of groups (ADHD with intervention vs. controls, $p=0.05$; ADHD without intervention vs. controls, $p=0.004$; the two ADHD groups, $p=0.34$ ). This two-way interaction however was better represented by a three-way interaction between preceding $\mathrm{FP}$, current $\mathrm{FP}$ and group $[F(8,168)=$ 3.59, $\left.p=0.0007, \eta_{\mathrm{p}}{ }^{2}=0.146\right]$. This interaction was mainly due to RTs for short FPs being more modulated by the preceding FP length for the two ADHD groups than for the healthy control group. To corroborate this hypothesis, planned comparisons were run with RT differences between the shortest and longest preceding FPs during the current short FP for each pair of groups. There was a significant difference between ADHD children with pharmacological intervention and healthy controls $(p=0.03)$ and ADHD children without intervention and healthy controls $(p=0.003)$, but not between the two ADHD groups $(p=0.43)$. No such difference was observed for current long FPs in any pair of groups (for all, $p>0.21$ ).

Another way to break the three-way interaction down was to look at the interaction between group and current FP at each preceding FP. No interaction between group and current FP was observed at the shortest preceding FP $(p=0.835)$. This interaction instead emerged for both the middle preceding FP $(p=0.0007)$ and the longest one $(p=0.0029)$. This analysis confirmed that the current FP effect of the two ADHD groups was more pronounced than that of controls only at longer preceding FPs.

A final way to gain insight into the preceding FP by current FP by group interaction is to unpack it by group. When we analyzed the data in this way, we found that 

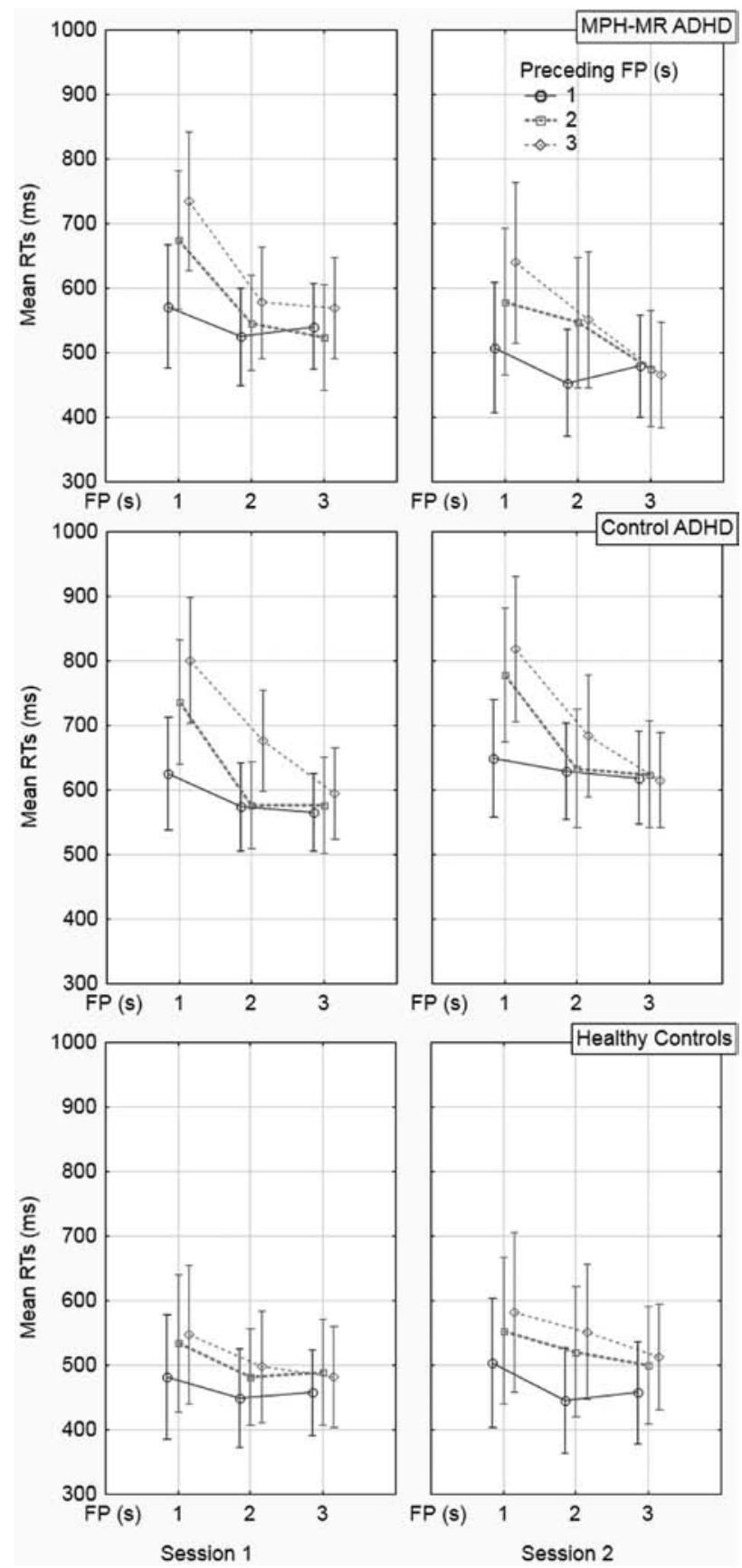

Figure 1. Mean RTs in ms (and 95\% confidence intervals) according to the three experimental groups, session, current foreperiod (FP) and preceding FP. 'MPH-MR ADHD' denotes the ADHD group with modified-release methylphenidate treatment; 'Control ADHD' indicates the control group of children with ADHD with no pharmacological treatment. This figure is published in colour in the online version. 
all groups showed a main effect of current FP (for all, $p<0.00015$ ) and preceding FP (for all, $p<0.00003$ ), demonstrating that all groups showed reliable variable FP effects and sequential effects. However, only the two ADHD groups showed a significant interaction between these two factors, that is, the asymmetric sequential effects (for both, $p<0.0002$ ), while the control group did not show this interaction $(p=0.52)$, demonstrating that sequential effects were present in this group (as shown by a preceding FP main effect) but symmetrically spread across the three current FPs. This finding replicates previously reported results (Vallesi \& Shallice, 2007), although with older children than those tested in the original study, and confirms that it is possible to dissociate the presence of the variable FP effect from the shape (symmetric vs. asymmetric) of the sequential effects (cf. Los \& Van den Heuvel, 2001).

There was also a significant session by group interaction $[F(2,42)=4.19$, $\left.p=0.022, \eta_{\mathrm{p}}{ }^{2}=0.17\right]$. This interaction seemed to be due to the fact that, while the ADHD group with pharmacological intervention decreased their RTs from the first session to the second one $(p=0.024)$, the other two groups numerically increased their RTs from one session to the next one (although this was not statistically significant, for both $p>0.15$ ). To back up this impression statistically, planned comparisons on RT differences between session 1 and 2 were run for each pair of samples. This analysis showed that ADHD children with pharmacological intervention were significantly different from healthy controls $(p=0.029)$ and ADHD children with no intervention $(p=0.009)$, while the other two groups did not show any difference $(p=0.72)$. If we look at these data from a different angle, in the first session RTs were longer for the ADHD group with no subsequent intervention than for healthy controls $(p<0.033)$ and tended to be higher for the ADHD group with subsequent treatment than for healthy controls $(p=0.09)$, with no difference between the two ADHD samples $(p=0.331)$. In the second session, however, the ADHD children after pharmacological intervention did not differ from healthy controls $(p=0.902)$ and outperformed ADHD children with no intervention $(p=0.017)$, while in the latter group the disadvantage with respect to the control group was maintained $(p=0.012)$. No other effect emerged as significant.

\subsection{Standard Deviation}

Standard Deviations (SD) are displayed in Fig. 2. There was a significant group main effect $\left[F(2,42)=3.82, p=0.0299, \eta_{\mathrm{p}}^{2}=0.15\right]$. Planned comparisons showed that the ADHD group with no intervention had larger SDs (collapsing the two sessions) than the other two groups $(p<0.001)$. Moreover, the ADHD group with treatment had larger SDs than healthy controls $(p<0.001)$. There were also preceding $\operatorname{FP}\left[F(2,84)=10.038, p=0.0001, \eta_{\mathrm{p}}^{22}=0.19\right]$ and current $\mathrm{FP}[F(2,84)=$ $\left.13.72, p<0.0001, \eta_{\mathrm{p}}^{2}=0.25\right]$ main effects. Importantly there was a significant by group interaction $\left[F(2,42)=4.06, p=0.0244, \eta_{\mathrm{p}}{ }^{2}=0.16\right]$. This interaction 

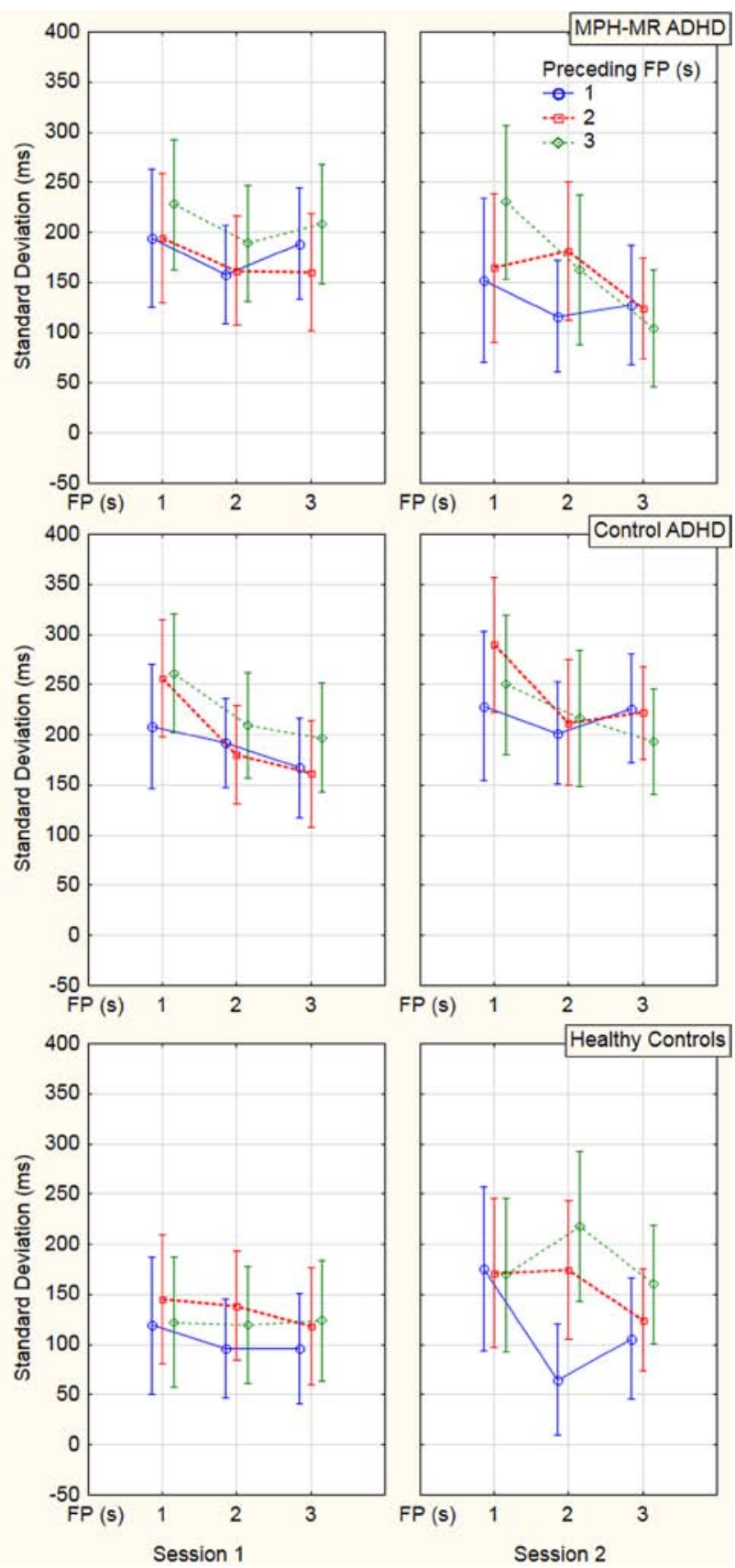

Figure 2. Standard deviation of response times (RTs) in ms (and 95\% confidence intervals) according to the three experimental groups, session, current foreperiod (FP) and preceding FP. See Fig. 1 for further details. This figure is published in colour in the online version. 
seemed to be due to the fact that while the ADHD children with pharmacological treatment tended to reduce their SDs from the first session to the second one $(p=0.059)$, the other two groups numerically increased their SDs from session 1 to session 2 (although this effect did not reach statistical significance, for both $p>0.09$ ). To corroborate this impression statistically, planned comparisons on SD differences between session 1 and 2 were run for each pair of samples. This analysis showed that the between-session SD difference in the ADHD children with pharmacological intervention was significantly different (i.e., higher) than the difference in healthy controls $(p=0.013)$ and ADHD children with no intervention $(p=0.022)$, while these two groups did not differ from each other $(p=0.73)$. To put it differently, while in the first baseline session SDs were higher for both ADHD groups than for healthy controls (for both, $p<0.033$ ), with no difference between each other $(p=0.57)$, in the second session, ADHD children after treatment reached the level of healthy controls $(p=0.99)$ and outperformed ADHD children with no intervention $(p=0.032)$, while the latter group showed larger SDs than the healthy controls $(p=0.031)$ as in the first session. No other effects emerged as significant.

\subsection{Early Responses (during FP and Soon after IS) and Slow Responses}

Anticipation responses during the FP and before the target onset occurred on 8\% of the trials and this percentage did not differ significantly across the three groups (one-way ANOVA, $p=0.36$ ). Early responses after target onset $(\mathrm{RT}<200 \mathrm{~ms}$ ) were rare (1.77\% of total responses) and did not differ across groups (one-way ANOVA, $p=0.33$ ). Delayed (RT $>2000 \mathrm{~ms}$ ) and null responses (2.27\% of total responses) also did not differ across groups (1-way ANOVA, $p=0.53$ ).

\section{Discussion}

This is the first study in which implicit temporal preparation was studied in ADHD with a variable FP paradigm as well as the first to assess the cognitive effects of MPH-MR in time preparation. Our results showed that children with ADHD had general performance deficits such as longer and more variable RTs with respect to a control group of age-matched controls. Notably, general RT slowness and variability significantly diminished in the experimental group after MPH-MR pharmacological treatment. Our findings confirmed the main conclusions of a recent quantitative review (Coghill et al., 2014), which demonstrated that MPHIR (mostly given as a single dose) improves various basic cognitive performance measures in ADHD, including RT speed and (reduced) variability (also see Kuntsi et al., 2003; Scheres et al., 2003; but see Johnson et al., 2008, for more specific effects of MPH therapy on indices of executive functioning), and extends these results to the continuous use of MPH-MR for one month.

The present study was also specifically designed to test how ADHD children implement temporal preparation by using a variable FP paradigm and how their 
performance on this task may change as a function of MPH-MR pharmacological treatment. This paradigm allows the dissociation of the automatic and controlled processes involved in temporal preparation (e.g., Steinborn \& Langner, 2011; Vallesi \& Shallice, 2007; Vallesi et al., 2014). Specific ADHD-related time preparation deficits included pronounced sequential FP effects at current short FPs, which suggests a higher susceptibility to automatic time-preparation processes (e.g., Los et al., 2014; Vallesi \& Shallice, 2007). This pattern of results did not improve with pharmacological treatment, suggesting that the MPH-MR effect does not specifically influence automatic time preparation processes.

Surprisingly, ADHD was not associated with a reduction of the variable FP effect, which according to some studies reflects more strategic time-preparation processes which include monitoring changing conditional probabilities in time by the lateral prefrontal cortex (e.g., Stuss et al., 2005; Vallesi et al., 2007a, b). On the contrary, the FP effect was exaggerated in the two ADHD groups as compared to controls, as shown by a significant current FP by group interaction. This may suggest that ADHD does not disrupt controlled monitoring processes in time preparation. There is instead the impression that these processes might even be enhanced in ADHD. However, it is possible that the variable FP effect, which is usually seen as a measure of controlled preparatory processes, may have been spuriously enhanced by a high influence of previous FP length at the shortest FPs, that is, by the presence of exaggerated sequential effects for the shortest current FPs. A follow-up Pearson's correlation analysis indeed showed that the latter could be the case, as the FP effect (calculated as the RT difference between the shortest and longest current FPs) and sequential effects on shortest FPs (calculated as the RT difference between the longest and shortest preceding FPs during the shortest current FPs) were positively correlated $(r=0.37, p=0.012)$, when the three groups and the two sessions were collapsed together to maximize statistical power (no significant effect emerged instead when the correlations were computed separately for each group). An alternative way to understand how the sequential effects might influence the current FP effect would be to look at the current FP effect at each previous FP. When the data were analyzed in this way, the FP effect was comparable across groups at the shortest previous FP, while group differences emerged at the middle and longest previous FPs, thus confirming that, in the present study, the estimated magnitude of the variable FP effect in ADHD children was probably confounded by the presence of exaggerated asymmetric sequential effects.

The limitations of the present study are the relatively small clinical sample of patients that does not allow generalization to the whole ADHD population, and the lack of a placebo control group with ADHD, which could have controlled for pharmacologically independent effects of treatment. Ethical considerations however prevented us from recruiting such a control group. Future studies should try to overcome this limit by recruiting different groups of ADHD children who may 
receive treatment at different time schedules, or by comparing the effect of different medication types in different ADHD groups.

In conclusion, this first investigation on the effect of MPH-MR on time preparation showed that ADHD is associated with worse performance in terms of absolute RTs and their variability. These deficits can be improved with MPH-MR pharmacological treatment. More ADHD-specific effects in implicit temporal preparation, such as exaggerated sequential effects with short preparatory intervals, are more resistant to MPH-MR intervention in ADHD, suggesting that this pharmacological therapy exerts its influence on general motor processing rather than on the more specific cognitive abilities involved in implicit time preparation.

\section{Acknowledgments}

AV was partially funded by the EU 7th Framework Programme European Research Council (ERC) starting grant LEX-MEA (GA\# 313692).

\section{References}

American Psychiatric Association (2000). Diagnostic and Statistical Manual of Mental Disorders. Fourth Edition, Text Revision American Psychiatric Association, Washington, DC, USA.

American Psychiatric Association (2013). Diagnostic and Statistical Manual of MentalDisorders. Fifth Edition. American Psychiatric Association; Washington, DC., USA http://dx.doi.org/10.1176/ appi.books.9780890425596.

Banaschewski, T., Coghill, D., Santosh, P., Zuddas, A., Asherson, P., Buitelaar, J., Danckaerts, M., Döpfner, M., Faraone, S. V., Rothenberger, A., Sergeant, J., Steinhausen, H. C., Sonuga-Barke, E. J., \& Taylor, E. (2006). Long-acting medications for the hyperkinetic disorders. A systematic review and European treatment guideline. Eur. Child Adolesc. Psychiatry, 15, 476-495.

Biancardi, A., \& Nicoletti C. (2004). Batteria per la discalculia evolutiva. Turin, Italy: Edizioni Omega. Carrer, L. R. J. (2015). Music and sound in time processing of children with ADHD. Front. Psychiatry, 6, 127. http://dx.doi.org/10.3389/fpsyt.2015.00127.

Casey, B. J., Castellanos, F. X., Giedd, J. N., Marsh, W. L., Hamburger, S. D., Schubert, A. B., Vauss, Y. C., Vaituzis, A. C., Dickstein, D. P., Sarfatti, S. E., \& Rapoport, J. L. (1997). Implication of right frontostriatal circuitry in response inhibition and attention-deficit/hyperactivity disorder.J. Am. Acad. Child Adolesc. Psychiatry, 36, 374-383.

Coghill, D. R., Seth, S., Pedroso, S., Usala, T., Currie, J., \& Gagliano, A. (2014). Effects of methylphenidate on cognitive functions in children and adolescents with attention-deficit/ hyperactivity disorder: Evidence from a systematic review and a meta-analysis. Biol. Psychiatry, $76,603-615$.

Conners, C. K. (2007). Conners' Parents and Teachers Rating Scales Revised. Adattamento italiano a cura di M. Nobile, B. Alberti \& A. Zuddas. Florence, Italy: Edizioni Organizzazioni Speciali.

Cubillo, A., Halari, R., Smith, A., Taylor, E., \& Rubia, K. (2012). A review of fronto-striatal and frontocortical brain abnormalities in children and adults with Attention Deficit Hyperactivity Disorder (ADHD) and new evidence for dysfunction in adults with ADHD during motivation and attention. Cortex, 48, 194-215. 
Curatolo, P., Paloscia, C., D’Agati, E., Moavero, R., \& Pasini, A. (2009). The neurobiology of attention deficit/hyperactivity disorder. Eur. J. Paediatr. Neurol., 13, 299-304.

Curatolo, P., D'Agati, E., \& Moavero, R. (2010). The neurobiological basis of ADHD. Ital. J. Pediatr., 36, 79. http://doi.org/10.1186/1824-7288-36-79.

González, M. A., Pentikis, H. S., Anderl, N., Benedict, M. F., DeCory, H. H., Dirksen, S. J. H., \& Hatch, S. J. (2002). Methylphenidate bioavailability from two extended-release formulations. Int. J. Clin. Pharmacol. Ther., 40, 175-184.

Hurks, P. P. M., Adam, J. J., Hendriksen, J. G. M., Vles, J. S. H., Feron, F. J. M., Kalff, A. C., Kroes, M., Steyaert, J., Crolla, I. F., Van Zeben, T. M., \& Jolles, J. (2005). Controlled visuomotor preparation deficits in attention-deficit/hyperactivity disorder. Neuropsychology, 19, 66-76.

Johnson, K. A., Barry, E., Bellgrove, M. A., Cox, M., Kelly, S. P., Dáibhis, A., Daly, M., Keavey, M., Watchorn, A., Fitzgerald, M., McNicholas, F., Kirley, A., Robertson, I. H., \& Gill, M. (2008). Dissociation in response to methylphenidate on response variability in a group of medication naïve children with ADHD. Neuropsychologia, 46, 1532-1541.

Kaufman, J., Brent, D., Rao, U., \& Ryan, N. (2004). K-SADS-PL. Intervista diagnostica per la valutazione dei disturbi psicopatologici in bambini ed adolescenti. Trento, Italy: Erickson.

Klein, C., Wendling, K., Huettner, P., Ruder, H., \& Peper, M. (2006). Intra-subject variability in attention-deficit hyperactivity disorder. Biol. Psychiatry, 60, 1088-1097.

Kramer, A. F., Cepeda, N. J., \& Cepeda, M. L. (2001). Methylphenidate effects on task-switching performance in attention-deficit/hyperactivity disorder. J. Am. Acad. Child Adolesc. Psychiatry, 40, $1277-1284$.

Kuntsi, J., Oosterlaan, J., \& Stevenson, J. (2003). Psychological mechanisms in hyperactivity: I. Response inhibition deficit, working memory impairment, delay aversion, or something else? J. Child Psychol. Psychiatry, 42, 199-210.

Leth-Steensen, C., Elbaz, Z. K., \& Douglas, V. I. (2000). Mean response times, variability, and skew in the responding of ADHD children: A response time distributional approach. Acta Psychol., 104, 167-190.

Los, S. A., \& Van den Heuvel, C. E. (2001). Intentional and unintentional contributions to nonspecific preparation during reaction time foreperiods. J. Exp. Psychol. Hum. Percept. Perform., 27, 370-386.

Los, S. A., Kruijne, W., \& Meeter, M. (2014). Outlines of a multiple trace theory of temporal preparation. Front. Psychol., 5, 1058. http://dx.doi.org/10.3389/fpsyg.2014.01058.

Luman, M., Van Noesel, S. J. P., Papanikolau, A., Van Oostenbruggen-Scheffer, J., Veugelers, D., Sergeant, J. A., \& Oosterlaan, J. (2009). Inhibition, reinforcement sensitivity and temporal information processing in ADHD and ADHD+ODD: Evidence of a separate entity? J. Abnorm. Child Psychol., 37, 1123-1135.

McDonald, S., Bennett, K. M., Chambers, H., \& Castiello, U. (1999). Covert orienting and focusing of attention in children with attention deficit hyperactivity disorder. Neuropsychologia, 37, 345-356.

NICE (2008). Attention deficit hyperactivity disorder - Guidance and guidelines. National Institute for Healthand Care Excellence.https://www.nice.org.uk/guidance/cg72/chapter/recommendations. Niemi, P., \& Näätänen, R. (1981). Foreperiod and simple reaction time. Psychol. Bull., 89, 133-162. Noreika, V., Falter, C. M., \& Rubia, K. (2013). Timing deficits in attention-deficit/hyperactivity disorder (ADHD): Evidence from neurocognitive and neuroimaging studies. Neuropsychologia, 51, 235-266. 
Oosterlaan, J., Scheres, A., \& Sergeant, J. A. (2005). Which executive functioning deficits are associated with AD/HD, ODD/CD and comorbid AD/HD+ODD/CD? J. Abnorm. Child Psychol., 33, 69-85.

Pelham, W. E., Gnagy, E. M., Burrows-Maclean, L., Williams, A., Fabiano, G. A., Morrisey, S. M., Chronis, A. M., Forehand, G. L., Nguyen, C. A., Hoffman, M. T., Lock, T. M., Fielbelkorn, K., Coles, E. K., Panahon, C. J., Steiner, R. L., Meichenbaum, D. L., Onyango, A. N., \& Morse, G. D. (2001). Once-a-day Concerta methylphenidate versus three-times-daily methylphenidate in laboratory and natural settings. Pediatrics, 107, E105.

Pelham, W. E., Waxmonsky, J. G., Schentag, J., Ballow, C. H., Panahon, C. J., Gnagy, E. M., Hoffman, M. T., Burrows-MacLean, L., Meichenbaum, D. L., Forehand, G. L., Fabiano, G. A., Tresco, K. E., Lopez-Williams, A., Coles, E. K., \& González, M. A. (2011). Efficacy of a methylphenidate transdermal system versus t.i.d. methylphenidate in a laboratory setting. J. Atten. Disord., 15, 28-35.

Perchet, C., Revol, O., Fourneret, P., Mauguière, F., \& Garcia-Larrea, L. (2001). Attention shifts and anticipatory mechanisms in hyperactive children: An ERP study using the Posner paradigm. Biol. Psychiatry, 50, 44-57.

Sartori, G., Job, R., \& Tressoldi, P. E. (1995). Batteria per la valutazione della dislessia e della disortografia evolutiva. Florence, Italy: Edizioni Organizzazioni Speciali.

Scheres, A., Oosterlaan, J., Swanson, J., Morein-Zamir, S., Meiran, N., Schut, H., Vlasveld, L., \& Sergeant, J. A. (2003). The effect of methylphenidate on three forms of response inhibition in boys with AD/HD. J. Abnorm. Child Psychol., 31, 105-120.

Schweitzer, J. B., Lee, D. O., Hanford, R. B., Zink, C. F., Ely, T. D., Tagamets, M. A., Hoffman, J. M., Grafton, S. T., \& Kilts, C. D. (2004). Effect of methylphenidate on executive functioning in adults with attention-deficit/hyperactivity disorder: Normalization of behavior but not related brain activity. Biol. Psychiatry, 56, 597-606.

Sergeant, J. A., Geurts, H., \& Oosterlaan, J. (2002). How specific is a deficit of executive functioning for attention-deficit/hyperactivity disorder? Behav. Brain Res., 130, 3-28.

Spencer, T. J., Biederman, J., Madras, B. K., Faraone, S. V., Dougherty, D. D., Bonab, A. A., \& Fischman, A. J. (2005). In vivo neuroreceptor imaging in attention-deficit/hyperactivity disorder: a focus on the dopamine transporter. Biol. Psychiatry, 57, 1293-1300.

Steinborn, M. B., \& Langner, R. (2011). Distraction by irrelevant sound during foreperiods selectively impairs temporal preparation. Acta Psychol., 136, 405-418.

Stuss, D. T., Alexander, M. P., Shallice, T., Picton, T. W., Binns, M. A., Macdonald, R., Borowiec, A., \& Katz, D. I. (2005). Multiple frontal systems controlling response speed. Neuropsychologia, 43, 396-417.

Taylor, E., Döpfner, M., Sergeant, J., Asherson, P., Banaschewski, T., Buitelaar, J., Coghill, D., Danckaerts, M., Rothenberger, A., Sonuga-Barke, E., Steinhausen, H. C., \& Zuddas, A. (2004). European clinical guidelines for hyperkinetic disorder - First upgrade. Eur. Child Adolesc. Psychiatry, 13 Suppl. 1, 17-30.

Toplak, M. E., Dockstader, C., \& Tannock, R. (2006). Temporal information processing in ADHD: findings to date and new methods. J. Neurosci. Meth., 151, 15-29.

Triviño, M., Correa, A., Arnedo, M., \& Lupiáñez, J. (2010). Temporal orienting deficit after prefrontal damage. Brain, 133, 1173-1185.

Vallesi, A., \& Shallice, T. (2007). Developmental dissociations of preparation over time: deconstructing the variable foreperiod phenomena. J. Exp. Psychol. Hum. Percept. Perform., 33, 1377-1388.

Vallesi, A., Mussoni, A., Mondani, M., Budai, R., Skrap, M., \& Shallice, T. (2007a). The neural basis of temporal preparation: Insights from brain tumor patients. Neuropsychologia, 45, 2755-2763. 
Vallesi, A., Shallice, T., \& Walsh, V. (2007b). Role of the prefrontal cortex in the foreperiod effect:TMS evidence for dual mechanisms in temporal preparation. Cereb. Cortex, 17, 466-474.

Vallesi, A., D’Agati, E., Pasini, A., Pitzianti, M., \& Curatolo, P. (2013a). Impairment in flexible regulation of speed and accuracy in children with ADHD. J. Int. Neuropsychol. Soc., 19, 1016-1020.

Vallesi, A., Lozano, V. N., \& Correa, A. (2013b). Dissociating temporal preparation processes as a function of the inter-trial interval duration. Cognition, 127, 22-30.

Vallesi, A., Arbula, S., \& Bernardis, P. (2014). Functional dissociations in temporal preparation: Evidence from dual-task performance. Cognition, 130, 141-151.

Woodrow, H. (1914). The measurement of attention. Psychol. Monogr., 17, 1-158. 